\title{
Effects of Perinatal Stress on the Emotion-Related Behavior of the Adolescent Mouse
}

\author{
Kohji Tokumo, Takao HiRai, and Hiroaki Nishino* \\ Department of Molecular Pharmacology, Faculty of Pharmacy and Pharmaceutical Sciences, Fukuyama University; \\ Hiroshima 729-0292, Japan. \\ Received December 29, 2005; accepted January 23, 2006; published online January 27, 2006
}

\begin{abstract}
We examined the effects of perinatal stress on the emotion-related behavior of the mouse during their adolescent period. Firstly, we applied the sound noise and/or the forced swim stress to the maternal pregnant mice in the pregnant mice at 10 to $18 \mathrm{~d}$ gestation. The stress applied during the fetal period did affect neither the spontaneous locomotor activity of the offspring mice in a new environment at an age of 4 weeks, nor those soundinduced changes in the locomotor activity. Secondly, we applied the stress to the neonatal mouse during the late lactation period, 14 to $18 \mathrm{~d}$ after birth. The mice that underwent the forced swim stress accompanied by the sound noise stress showed a significant reduction of the locomotor activity. On the other hand, the mice that underwent the forced swim stress during the lactation period showed a significant increase in the locomotor activity after the stimulation by the sound noise. Lastly, we applied the stress to the neonatal mouse immediately after the weaning period, 21 to $25 \mathrm{~d}$ after birth. The spontaneous locomotor activity of the mice was not changed compared to that of the non-stressed group. Thus, it was found that the stress applied to the mice during the lactation period, thought to be the critical period of mouse brain development, selectively influenced the emotion-related behavior in the subsequent adolescent period. These results suggested that second postnatal week may be the critical period for establishing proper behavioral responses to emotional stress in the adolescent mouse.
\end{abstract}

Key words stress; prenatal; postnatal; emotional behavior; sound noise; forced swim

There are a large variety of stresses in the environment surrounding the pregnant mother and infant. Several lines of evidence have shown that stressful life events increase the susceptibility to emotional stress and contribute to the development of major depression or posttraumatic stress disorders (PTSD) ${ }^{1-3)}$ Early life stresses precipitate long-lasting alteration in the functional properties underlying emotional expression and may consequently alter the responses to stress later in adolescent life. Exposure to environmental noise may affect the fetus as well as the pregnant woman. ${ }^{4)}$ It is generally accepted that the fetus in pregnant woman experiences the sound of the mother's blood stream. In late gestation, the fetus is capable of hearing sound from outside the woman. The acoustic stimuli reaching the inner ear of the fetus are strong enough to excite the fetus and cause an acceleration of heart rate and body movement, providing evidence of discomfort. ${ }^{5)}$ Mental and physical healthcare of pregnant women and infants might affect the normal development of the fetus through the effects on the synaptogensis in the brain. ${ }^{6}$

In the present study, we applied stresses with the sound noise and/or forced swim to pregnant mice or neonatal mice, and examined their effects on the emotion-related behavior of the mice during the adolescent period.

\section{MATERIALS AND METHODS}

Pregnant ddY mice were obtained from Japan SLC. The experimental animals received food (CE-2, Clea Japan, Inc.) and tap water ad libitum, and were housed under standard laboratory conditions (room temperature $23 \pm 1^{\circ} \mathrm{C}$, humidity 55 $\pm 5 \%$, lights on 07:00 - 19:00). The Guidelines of Ethical Standards for Animal Experiments of the Fukuyama University were followed.
Prenatal Stress during the Fetal Period On day 10 of pregnancy, the pregnant mice were divided into 4 groups, namely pregnant mouse [dam-N], [dam-SO], [dam-SW] and [dam-SS], and they were manipulated as described below. Five mice were used in each group. The [dam-SO] group was exposed to the $90 \mathrm{~dB}$ sound noise every minute for $15 \mathrm{~min}$ once a day from day 10 to 18 of pregnancy. The neonatal mice from the [dam-SO] were referred to as [pre-SO]. The [dam-SW] group was forced to swim for 15 min once a day from day 10 to 18 of pregnancy. The neonatal mice from the [dam-SW] were referred to as [pre-SW]. The [dam-SS] group was forced to swim for 15 min once a day accompanied by the sound noise stress from day 10 to 18 of pregnancy. The neonatal mice from the [dam-SS] were designated [pre-SS]. No stress manipulation was applied to the pregnant mouse [dam-N]. The neonatal male mice from [dam-N] were called [pre-N]. All neonatal mice were reared by each dam under the standard laboratory conditions.

Postnatal Stress during the Lactation Period Fourteen days after birth, the neonatal mice were divided into 4 groups, namely [dl-N], [dl-SO], [dl-SW] and [dl-SS], and they were manipulated as described below. The [dl-SO] group was exposed to the $90 \mathrm{~dB}$ sound every minute for $15 \mathrm{~min}$ once a day from 14 to $18 \mathrm{~d}$ after birth. The [dl-SW] group was forced to swim for $15 \mathrm{~min}$ once a day from 14 to $18 \mathrm{~d}$ after birth. The [dl-SS] group was forced to swim for 15 min once a day, accompanied by the sound noise stress from day 10 to 18 of pregnancy. No stress manipulation was applied to the mouse [dl-N].

Postnatal Stress Immediately after the Weaning Period The neonatal mice were weaned at $21 \mathrm{~d}$ after birth. They were divided into 4 groups, namely [aw-N], [aw-SO], [aw$\mathrm{SW}]$ and [aw-SS], and they were manipulated as described below. The [aw-SO] group was exposed to the $90 \mathrm{~dB}$ sound 


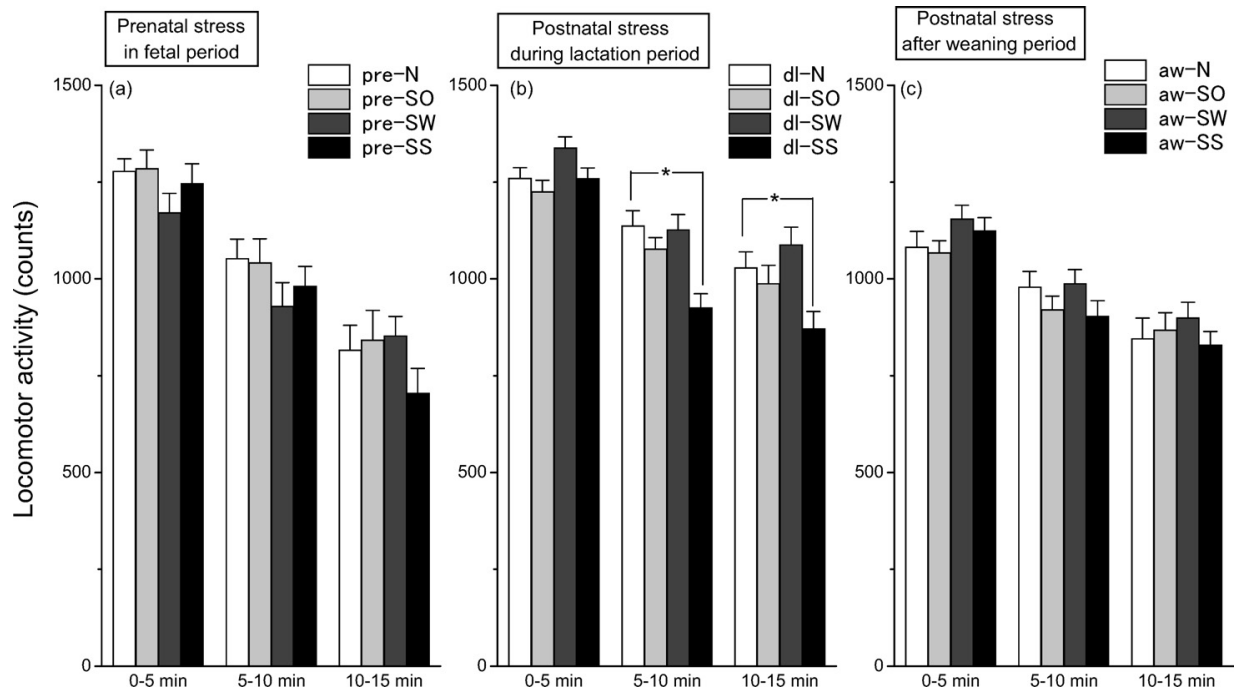

$\dagger$ Fig. 1. Effects of the Prenatal Stress in the Fetal Period, the Postnatal Stress during the Lactation Period and the Postnatal Stress on the Locomotor Activities of the Mouse Observed at an Age of 4 Weeks

The sound noise and/or forced swim stress was applied according to the procedure described in the text. After a 5 min acclimation period, the locomotor activity was measured at $5 \mathrm{~min}$ intervals for three sessions, expressed as $0-5 \mathrm{~min}, 5-10 \mathrm{~min}$ and $10-15 \mathrm{~min}$. Data are from 33 to 42 animals in each group. Significance: $* p<0.001$.

noise every minute for $15 \mathrm{~min}$ once a day from 21 to $25 \mathrm{~d}$ after birth. The [aw-SW] group was forced to swim for 15 min once a day from 21 to $25 \mathrm{~d}$ after birth. The [aw-SS] group was forced to swim for $15 \mathrm{~min}$ once a day accompanied by the sound noise stress from day 10 to 18 of pregnancy. No stress manipulation was applied to the mouse [aw-N].

At an age of 4 weeks, the locomotor activity of the manipulated mouse was quantified using an activity sensor (NAAS01, Neuroscience, Inc., Japan). The activity was measured every $5 \mathrm{~min}$ successively for $25 \mathrm{~min}$. Then, the $90 \mathrm{~dB}$ sound noise for $20 \mathrm{~s}$ was applied after the session of $10-15 \mathrm{~min}$. The effect of sound noise stimulation was estimated by calculating the percentage of activity in the sessions of 15 $25 \mathrm{~min}$, taking the activity in the sessions of $5-15 \mathrm{~min}$ as $100 \%$.

For the analysis of behavioral data, repeated measures oneway ANOVAs and Student's $t$-tests for independent samples were used. The $p$-values given are two-tailed, and represent measures of effect.

\section{RESULTS}

The sound noise and/or forced swim stress were applied to the pregnant maternal mouse or postnatal mouse. During the adolescent period, 4 weeks after birth, the emotion-related behavior of the mouse was examined. The spontaneous locomotion activity of mice in the new environment was gradually reduced along with the session of measurements (Fig. 1). The activity of the mice which underwent stress in the late pregnant period, [pre-SO], [pre-SW] and [pre-SS], did not change from that of non-stressed group, [pre-N] (Fig. 1a). The activity of the neonatal mice that underwent the sound noise or forced swim stress in the lactation period, [dl-SO] and [dl-SW], did not change from that of non-stressed group, [dl-N] (Fig. 1b). However, the mice that underwent the forced swim stress accompanied by the sound noise stress, [dl-SS], showed a significant reduction of the locomotor ac-

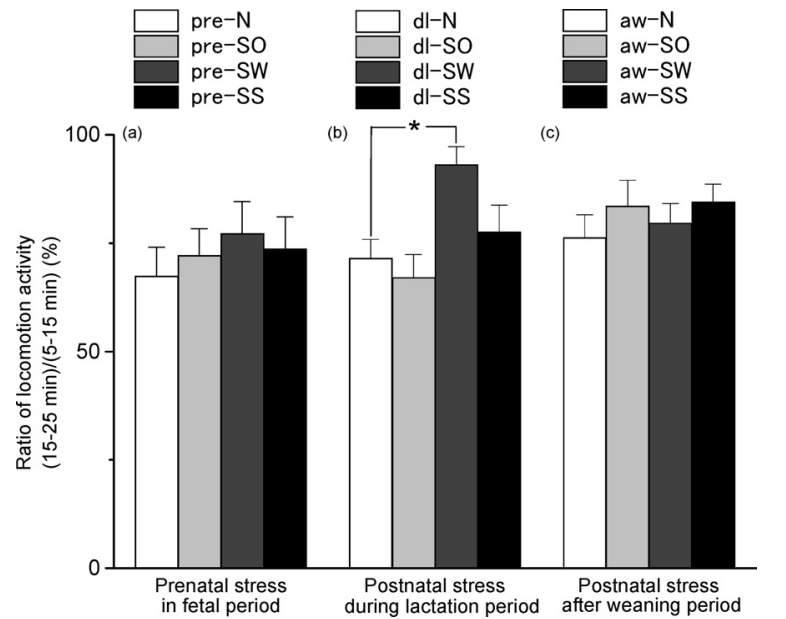

Fig. 2. Effects of the Prenatal Stress in the Fetal Period, the Postnatal Stress during the Lactation Period and the Postnatal Stress on the Ratio of Locomotor Activities beteen Pre- and Post-sound Noise Stimulation

The sound noise and/or forced swim stress was applied according to the procedure described in the text. After a $10-15 \mathrm{~min}$ session, the $90 \mathrm{~dB}$ sound noise stimulation was applied for $20 \mathrm{~s}$. The ratio of locomotor activity between the locomotor activity in $15-25 \mathrm{~min}$ and 5-15 min sessions was calculated. Data are from 33 to 42 animals in each group. Significance: $* p<0.001$.

tivity during the $5-10 \mathrm{~min}$ and $10-15 \mathrm{~min}$ sessions (Fig. 1b). On the other hand, the spontaneous locomotor activity of the mice that underwent the postnatal stress immediately after the weaning period, [aw-SO], [aw-SW] and [aw-SS], did not change from that of non-stressed group, [aw-N] (Fig. 1c).

Figure 2 illustrates the change in the spontaneous locomotor activity following the stimulation by the sound noise applied at $15 \mathrm{~min}$ after the measurement. Among the groups of stress mice, only the [dl-SW] mice, which underwent the forced swim stress in the lactation period, showed a significant increase in the locomotor activity compare to that observed in the non-stressed mice [dl-N] (Fig. 2). 


\section{DISCUSSION}

In the present study, the effects of perinatal stress on the emotion-related behavior of the mouse at the adolescent period were evaluated. We applied the sound noise and/or forced swim stress on the fetal mice, the neonatal mice during the lactation period and immediately after the weaning period, respectively. The spontaneous locomotion activity of the adolescent mouse in a new environment and the change in the locomotor activity following the stimulation by sound noise were measured. When the stresses were applied to the fetal mice, there were no changes in the emotion-related behavior of the mouse observed during the adolescent period. We have reported that the sound noise and forced swim stress, applied to pregnant rats, induced changes in the emotional behavior and learning ability of the neonatal rats. ${ }^{7}$ This disparity between mice and rats might be explained by the suggestion that the critical period of the brain functional development is dissimilar between these animals. ${ }^{8)}$

In conformity to these experimental results, we evaluated the effects of postnatal stress to observe the possible influence of stress on the mouse brain functional development. We applied the sound noise and/or forced swim stress to the neonatal mouse during the late lactation period, 14 to $18 \mathrm{~d}$ after birth. The results showed that the locomotor activity in the 5-10 $\mathrm{min}$ and $10-15 \mathrm{~min}$ sessions were significantly reduced by the forced swim stress accompanied by the sound noise stress. The mice that underwent the sound noise or the forced swim stress alone did not show any changes in their activity. These results suggested that the combined stresses during the lactation period of the mouse were strong enough to cause an adjustment disorder to a new environment. Furthermore, the mouse that underwent the forced swim stress during the lactation period showed a significant increase in the locomotor activity following the stimulation by the sound noise applied $15 \mathrm{~min}$ after the measurement. The result suggested that the forced swim stress applied during the lactation period, which showed no effect on the spontaneous locomotor activity, might provoke an excessive response to the application of the additional sound noise. However, the sound noise stressed mouse with or without forced swim stress did not respond to the application of the additional sound. These results might indicate that the mice that experienced the sound noise stress during lactation period showed the acclimation to the additional sound noise. Thus, it was found that the stress applied in the lactation period, which is supposed to be the critical period of mouse brain development, caused influence in its subsequent developmental process. These results were comparable to those which we found in the effects of prenatal stress applied to the pregnant rats on the emotional behavior and learning ability of offspring. ${ }^{7)}$

It is interesting to note that when the stress was applied immediately after weaning, 3 weeks after birth, the mice did not show any changes in the emotion-related behavior during the adolescent period. Lately, Matsumoto et al. ${ }^{9)}$ reported the alteration of the neuronal modulation to emotional stress by early postnatal stress during the postadolescent periods of rats. They showed that fear-related freezing behavior observed during exposure to contextual fear conditioning was markedly attenuated in the mouse exposed to aversive stimulus (foot-shock) at the postnatal period of the second week, but not of the third week. The second postnatal week may be the "critical period" for establishing proper behavioral responses to emotional stress in adult rats (Matsumoto et al., 2005). Our present results suggested that the "critical period" should be envisioned for mice, involving the adaptation to emotional stress in the adolescent mice. The effects of the stress applied in the critical period of mouse brain development on the emotional behavior and the learning behavior should be investigated in more detail, as well as those on the neurochemical parameters of the brain.

\section{REFERENCES}

1) McAllister-Williams R. H., Ferrier I. N., Young A. H., Psychol. Med., 28, 573-584 (1998).

2) Modell S., Lauer C. J., Schreiber W., Huber J., Krieg J. C., Holsboer F., Neuropsychopharmacol., 18, 253-262 (1998).

3) Heim C., Nemeroff C. B., Biol. Psychiatry, 49, 1023-1039 (2001).

4) Shiota K., Kayamura T., Biol. Neonate, 56, 6-14 (1989).

5) Szmeja Z., Slomko Z., Sikorski K., Sowinski H., Int. J. Pediatr. Otorhinolaryngol., 1, 221-229 (1979).

6) Hayashi A., Nagaoka M., Yamada K., Ichitani Y., Miake Y., Okado N., Int. J. Dev. Neurosci., 16, 209-216 (1998).

7) Nishio H., Kasuga S., Ushijima M., Harada Y., Int. J. Develop. Neurosci., 19, 37-45 (2001).

8) Arai Y., Tanpakushitsu Kakusan Koso, 35, 426 - 433 (1990) (in Japanese).

9) Matsumoto M., Higuchi K., Togashi H., Koseki H., Yamaguchi T., Kanno M., Yoshioka M., Hippocampus, 15, 775-781 (2005). 